Published in "Physical Review E 77(5): 021920, 2008"

which should be cited to refer to this work.

\title{
Singularities and symmetry breaking in swarms
}

\author{
Wei Li \\ Department of Automation, Shanghai Jiao Tong University, Shanghai, 200240, People's Republic of China \\ Hai-Tao Zhang \\ Department of Control Science and Engineering, Huazhong University \\ mof Science and Technology, Wuhan 430074, People's Republic of China \\ and Department of Engineering, University of Cambridge, Cambridge CB2 1PZ, United Kingdom \\ Michael ZhiQiang Chen* \\ Department of Engineering, University of Leicester, Leicester LE1 7RH, United Kingdom \\ Tao Zhou \\ Department of Modern Physics, University of Science and Technology of China, Hefei 230026, People's Republic of China \\ and Department of Physics, University of Fribourg, Chemin du Muse 3, CH-1700 Fribourg, Switzerland
}

\begin{abstract}
A large-scale system consisting of self-propelled particles, moving under the directional alignment rule (DAR), can often self-organize to an ordered state that emerges from an initially rotationally symmetric configuration. It is commonly accepted that the DAR, which leads to effective long-range interactions, is the underlying mechanism contributing to the collective motion. However, in this paper, we demonstrate that a swarm under the DAR has unperceived and inherent singularities. Furthermore, we show that the compelled symmetry-breaking effects at or near the singularities, as well as the topological connectivity of the swarm in the evolution process, contribute fundamentally to the emergence of the collective behavior; and the elimination or weakening of singularities in the DAR will induce an unexpected sharp transition from coherent movement to isotropic dispersion. These results provide some insights into the fundamental issue of collective dynamics: What is the underlying mechanism causing the spontaneous symmetry breaking and leading to eventual coherent motion?
\end{abstract}

PACS number(s): 87.10.-e

\section{INTRODUCTION}

The emergence of collective motions in biological swarms, such as schools of fish, flocks of birds, and colonies of bacteria, has been extensively observed in nature and in artificial simulations [1-11]. These collective biological groups can self-organize and travel as if they were an individual living creature. These complex and nonintuitive aggregated behaviors can be induced by a simple mechanism, namely, doing what the near neighbors do [1]. Common features of these phenomena are (1) no leader(s) or central control; (2) absence of external stimuli; (3) no global information sharing; and (4) homogeneous agents. Swarm behaviors have attracted increasing interest in many fields as they can provide insight into problems such as the collective motion control of robots $[12,13]$, human collective behaviors [14-16], material shape matters [17,18], and even the development of software agents in particle swarm optimization algorithms [19] and genetic algorithms [20].

In 1995, Vicsek et al. [21] proposed a novel model to imitate a biological swarm using self-propelled particles. This minimal model captures the important rule of directional alignment: every agent moves toward the average movement direction of its neighbors. The Vicsek model

\footnotetext{
*mzqchen@gmail.com
}

(VM) has been intensively investigated in recent years both theoretically [12,22-25] and, more recently, experimentally [7]. Compared to the constant-speed VM, a further extension can be addressed by the adaptive velocity strategy [26], whose basic idea is that each agent moves along the average direction of its neighbors but with adaptive speed-when an agent finds itself surrounded by scattered moving agents, it may naturally feel at a loss and thus move at a very slow speed; while if a certain direction of movement is dominant in its neighborhood, the agent tends to take this direction with a faster speed.

In this paper, we study swarms consisting of homogeneous agents that simply imitate what their near neighbors do, in particular the VM and the adaptive velocity model $(\mathrm{AVM})$ that capture the directional alignment rule (DAR). In such models, the system can often evolve from a rotationally symmetric state (agents' initial directions are uniformly distributed without any statistically dominant direction) to form one ordered congregation. One important feature is that the initial random distribution of agents' movement directions does not favor any predefined direction, and under the DAR (with or without adaptive speed), every agent takes the average direction of its near neighbors, which also has no preferential direction. No agent knows its destination, and the emergence of the collective swarm is a purely spontaneous result of all the agents' interactions. Naturally, there are some fundamental open questions regarding the emergence of order in such systems: Why does this DAR induce large-scale 


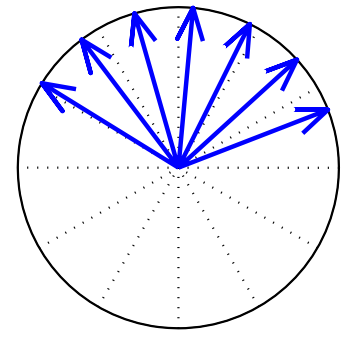

(a)

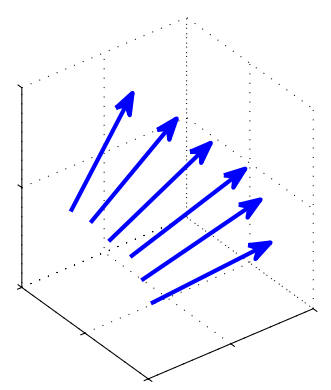

(c)

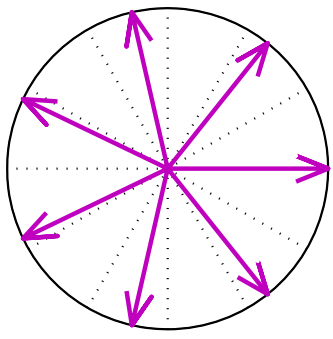

(b)

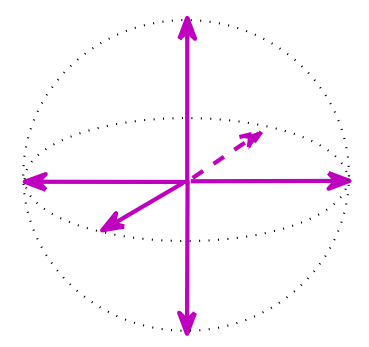

(d)
FIG. 1. (Color online) Illustration of the initial directional vectors of the swarm agents in 2D and 3D space. The arrows denote the unitary direction vectors. (a),(b) 2D and (c),(d) 3D case. (a),(c) Polar and (b),(d) isotropic cases. For simplicity, in each of the subfigures (a), (b), and (d), the modular vectors are plotted with the same starting point located at the origin of the coordinates.

emergence from the isotropy in the initial state? What factors contribute to such emergence? Can the emergent direction be predicted and is it robust to minor disturbances?

A popular interpretation of the emergence of order is that agents are able to move and mix in the system. This mixture can result in effective long-range interactions among agents, which induces a phase transition from initial disorder to order [21,27-29]. Indeed, the long-range interactions can serve as an explanation of the collective movement in polar cases such as Figs. 1(a) and 1(c), but it is insufficient to satisfactorily interpret the emergent order from the isotropic cases such as Figs. 1(b) and 1(d). Theoretical analyses [12,13,30] show that the emergence depends on the connectivity of graph topologies of the swarms, regardless of short-range or long-range interactions. However, we should note that (i) the existing literature $[12,13,30]$ does not consider actual topologies formed by the neighborhood relations of agents' positions in the evolutional process, and the connectivity property imposed on the system is only a hypothetical condition for the directional convergence result; and (ii) most importantly, although the DAR is symmetric, the literature only considers the linearity or quasilinearity of the DAR which implies predefined and predicted direction(s) of emergence of the collective swarm (refer to Appendixes A and B). However, the models with this (quasi)linearity eliminate the inherent nonlinearity properties of the swarms and cannot be viewed as rotationally symmetric any longer.

In this paper, we reveal the inherent singularities in the DAR of the swarm, which fundamentally contribute to the compelled symmetry-breaking effects. The elimination or weakening of such singularities tends to disperse the swarm. The emergent direction of a swarm also has the property of unpredictability. This article is organized as follows. Section II gives a brief review of the VM and AVM, which effectively embody the DAR. Section III illustrates the existence of singularities in the DAR of the models. The contribution of compelled symmetry-breaking effects at singularities to large-scale emergence is discussed in Sec. IV, together with the property of the unpredictability of the emergent direction. Our conclusions are given in the last section.

\section{BRIEF REVIEW OF VM AND AVM}

The VM supposes that all the agents move simultaneously with the same fixed speed $v_{0}$ updated at time steps $\Delta t=1$. At each time step, each agent assumes the average direction of the agents within its neighborhood of radius $R$. Let $\vec{X}_{i}(k)$ $\in \mathrm{C}$ denote the complex position vector of agent $i$ on the complex two-dimensional (2D) plane at time step $k$. Agent $i$ and agent $j$ are neighbors at time step $k$ if and only if the distance $\left|\vec{X}_{i}(k)-\vec{X}_{j}(k)\right| \leq R$, where $|\cdot|$ denotes the absolute value or modulus of a complex number. The constant speed VM can be described as follows:

$$
\begin{gathered}
\vec{X}_{i}(k+1)=\vec{X}_{i}(k)+v_{i}(k) \Delta t, \\
\theta_{i}(k+1)=\left\langle\theta_{i}(k)\right\rangle_{r},
\end{gathered}
$$

where $v_{i}(k)$ is the velocity of agent $i$, with its constant speed $\left|v_{i}(k)\right| \equiv v_{0}$. The notation $\left\langle\theta_{i}(k)\right\rangle_{r}$ denotes the average direction of neighbors within the neighborhood radius (including the agent itself). Here, the external noise presented in the original VM is not considered. The average direction is computed by the following equation [21]:

$$
\left\langle\theta_{i}(k)\right\rangle_{r}=\arctan \left(\frac{\left\langle\sin \left(\theta_{i}(k)\right)\right\rangle_{r}}{\left\langle\cos \left(\theta_{i}(k)\right)\right\rangle_{r}}\right) .
$$

The AVM [26] further extends the constant-speed VM by introducing the complex-valued local order parameter. Each agent adjusts not only its movement direction, but also its speed, in an adaptive fashion according to the degree of direction consensus among its neighbors. Let $\Gamma_{i}(k)$ be the set of agent $i$ 's neighbors (including $i$ itself) at time step $k$, and $n_{i}(k)$ the number of elements in $\Gamma_{i}(k)$. The AVM is then described as follows:

$$
\begin{gathered}
\vec{X}_{i}(k+1)=\vec{X}_{i}(k)+\phi_{i}^{\alpha}(k) e^{i \theta_{i}(k)} v_{0} \Delta t, \\
\phi_{i}(k+1) e^{i \theta_{i}(k+1)}=\frac{1}{n_{i}(k+1)} \sum_{j \in \Gamma_{i}(k+1)} e^{i \theta_{j}(k)}, \\
i=1,2, \ldots, N ; \quad k=0,1,2, \ldots,
\end{gathered}
$$

where $e^{i \theta_{j}(k)} \in \mathrm{C}$ is the unitary complex vector that represents the vectorial direction of agent $j$ at time step $k . \phi_{i}(k+1)$ measures the local degree of direction consensus among $i$ 's 

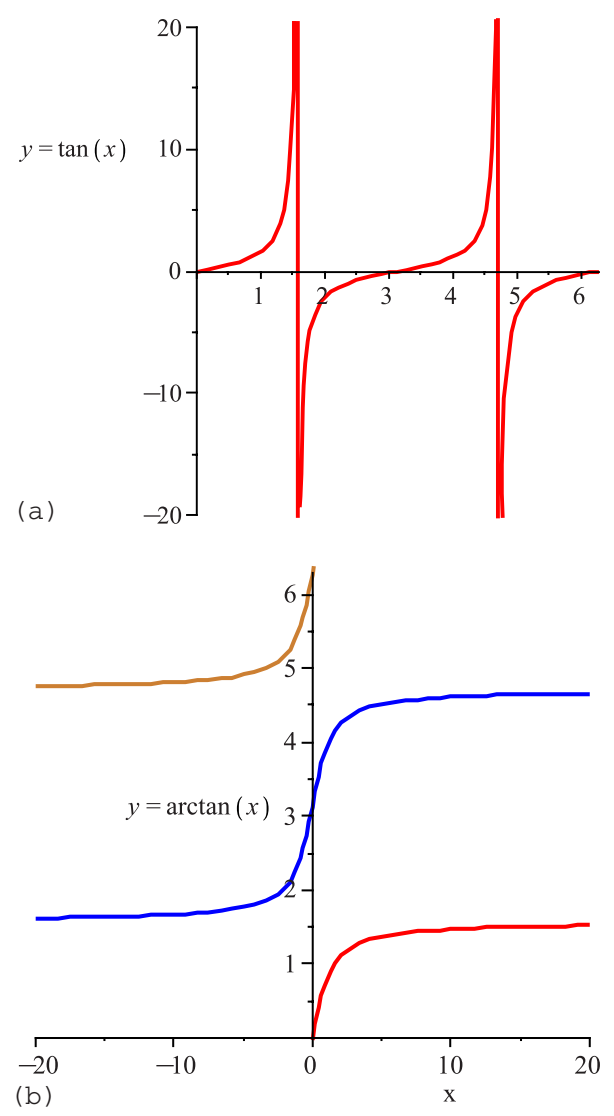

FIG. 2. (Color online) (a) Illustration of the tangent function $y$ $=\tan (x)$ on the domain $x \in[0,2 \pi)$ with the range $y \in(-\infty, \infty)$. (b) Illustration of the tangent function $y=\arctan (x)$ on the domain $x$ $\in(-\infty, \infty)$ with the range $y \in[0,2 \pi)$. The tangent function should be defined as a multivalued function in this context instead of any monotropic function. Actually, it has three overlapping branches as illustrated in (b).

neighbors at time step $(k+1)$. A larger $\phi_{i}(k+1)$ corresponds to better direction consensus. The angle $\theta_{i}(k+1)$ implied in Eq. (3),

$$
\theta_{i}(k+1)=\operatorname{angle}\left(\sum_{j \in \Gamma_{i}(k+1)} e^{i \theta_{j}(k)}\right)
$$

is the movement direction of agent $i$ at time step $(k+1)$, where the function angle(.) returns the angle of a com- plex number. $\vec{v}_{i}(k) \triangleq v_{0} \phi_{i}^{\alpha}(k) e^{i \theta_{i}(k)}$ represents the velocity of agent $i$ at time step $k$ with adaptive speed $\left|\vec{v}_{i}(k)\right|$ $=v_{0} \phi_{i}^{\alpha}(k)$ and direction $\theta_{i}(k)$. Since $0 \leq \phi_{i}(k) \leq 1$, the exponent $\alpha \geq 0$ just reflects the willingness of each agent to move faster or slower along the average direction of its neighbors based on the local degree of direction consensus. If $\alpha=0$, then $\phi_{i}^{\alpha}(k) \equiv 1$, and the AVM degenerates to the VM with each agent moving at the maximum speed $v_{0}$ without any consideration of its local polarity. The AVM with $\alpha>0$ induces a more intensified phase transition from a disordered to an ordered state compared with the VM [26].

\section{SINGULARITIES IN THE DAR}

The DAR should be rotationally symmetric, which is very different from the linearity or quasilinearity rules that actually prefer a certain movement direction (see Appendixes A and $\mathrm{B}$ for details). Next, we demonstrate the existence of singularities and the strongly nonlinear nature of the DAR in the swarm. Note that the parameter $\phi_{i}(k+1) e^{i \theta_{i}(k+1)}$ is expressed as the sum of complex unit vectors $e^{i \theta_{j}(k)}$ in Eq. (3). It can be equivalently expressed as the triangular formations:

$$
\begin{aligned}
\phi_{i}(k+1) \cos \left(\theta_{i}(k+1)\right) & =\frac{1}{n_{i}(k+1)} \sum_{j \in \Gamma_{i}(k+1)} \cos \left(\theta_{j}(k)\right), \\
\phi_{i}(k+1) \sin \left(\theta_{i}(k+1)\right) & =\frac{1}{n_{i}(k+1)} \sum_{j \in \Gamma_{i}(k+1)} \sin \left(\theta_{j}(k)\right)
\end{aligned}
$$

for all $i=1,2, \ldots, N$. Therefore, we have

$$
\theta_{i}(k+1)=\arctan \frac{\sum_{j \in \Gamma_{i}(k+1)} \sin \left(\theta_{j}(k)\right)}{\sum_{j \in \Gamma_{i}(k+1)} \cos \left(\theta_{j}(k)\right)} .
$$

Note that, in Eq. (5), the range of the arctangent function $\arctan (\cdot)$ or the domain of the tangent function $\tan (\cdot)$ should be defined in the interval $[0,2 \pi$ ) (see Fig. 2) instead of its principal value interval $(-\pi / 2, \pi / 2)$ as in Ref. [13] (see also Appendix B).

Considering that every agent will move toward the direction of its local polarity, it is natural that $\theta_{i}(k+1)$ in Eq. (5) should be defined unambiguously in the following multivalued function:

$$
\theta_{i}(k+1) \in\left\{\begin{array}{lcc}
\left(0, \frac{\pi}{2}\right), & \sum_{j \in \Gamma_{i}(k+1)} \sin \left(\theta_{j}(k)\right)>0, & \sum_{j \in \Gamma_{i}(k+1)} \cos \left(\theta_{j}(k)\right)>0, \\
\left(\frac{\pi}{2}, \pi\right), & \sum_{j \in \Gamma_{i}(k+1)} \sin \left(\theta_{j}(k)\right)>0, & \sum_{j \in \Gamma_{i}(k+1)} \cos \left(\theta_{j}(k)\right)<0, \\
\left(\pi, \frac{3 \pi}{2}\right), & \sum_{j \in \Gamma_{i}(k+1)} \sin \left(\theta_{j}(k)\right)<0, & \sum_{j \in \Gamma_{i}(k+1)} \cos \left(\theta_{j}(k)\right)<0, \\
\left(\frac{3 \pi}{2}, 2 \pi\right), & \sum_{j \in \Gamma_{i}(k+1)} \sin \left(\theta_{j}(k)\right)<0, & \sum_{j \in \Gamma_{i}(k+1)} \cos \left(\theta_{j}(k)\right)>0,
\end{array}\right.
$$




$$
\theta_{i}(k+1)=\left\{\begin{array}{lcc}
0, & \sum_{j \in \Gamma_{i}(k+1)} \sin \left(\theta_{j}(k)\right)=0, & \sum_{j \in \Gamma_{i}(k+1)} \cos \left(\theta_{j}(k)\right)>0, \\
\pi, & \sum_{j \in \Gamma_{i}(k+1)} \sin \left(\theta_{j}(k)\right)=0, & \sum_{j \in \Gamma_{i}(k+1)} \cos \left(\theta_{j}(k)\right)<0, \\
\frac{\pi}{2}, & \sum_{j \in \Gamma_{i}(k+1)} \sin \left(\theta_{j}(k)\right)>0, & \sum_{j \in \Gamma_{i}(k+1)} \cos \left(\theta_{j}(k)\right)=0, \\
\frac{3 \pi}{2}, & \sum_{j \in \Gamma_{i}(k+1)} \sin \left(\theta_{j}(k)\right)<0, & \sum_{j \in \Gamma_{i}(k+1)} \cos \left(\theta_{j}(k)\right)=0 .
\end{array}\right.
$$

Here $\theta_{i}(k+1) \in[0,2 \pi)$. Note that there is no difference between defining $\theta_{i}(k+1)=0$ or $2 \pi$ in the case of

$$
\sum_{j \in \Gamma_{i}(k+1)} \sin \left(\theta_{j}(k)\right)=0, \quad \sum_{j \in \Gamma_{i}(k+1)} \cos \left(\theta_{j}(k)\right)>0
$$

in Eq. (7); that is, the range interval of $\theta_{i}(k+1) \in[0,2 \pi)$ or $(0,2 \pi]$ has no effect on the behavior of the swarm, while with the linearity rule, the intervals $[0,2 \pi)$ and $(0,2 \pi]$ will lead to different behaviors in some extreme cases [31].

The dilemma of the DAR is that when

$$
\sum_{j \in \Gamma_{i}(k+1)} \sin \left(\theta_{j}(k)\right)=0, \quad \sum_{j \in \Gamma_{i}(k+1)} \cos \left(\theta_{j}(k)\right)=0
$$

the direction $\theta_{i}(k+1)$ cannot be defined, since

$$
\theta_{i}(k+1)=\arctan (0 / 0)=\text { undefined } .
$$

Actually, this is the inherent and unavoidable singularity in the DAR, which is equivalent to the expression $\phi_{i}(k+1)=0$ in Eq. (3) of the AVM, i.e.,

$$
\phi_{i}(k+1)=0 \Leftrightarrow\left\{\begin{array}{c}
\sum_{j \in \Gamma_{i}(k+1)} \sin \left(\theta_{j}(k)\right)=0, \\
\sum_{j \in \Gamma_{i}(k+1)} \cos \left(\theta_{j}(k)\right)=0 .
\end{array}\right.
$$

As mentioned above, $\phi_{i}(k+1)$ measures the local degree of direction consensus among the neighbors of agent $i$. If agent $i$ is in the apparently polar case [i.e., $\phi_{i}(k+1) \gg 0$ ], the average direction is actually well defined. For example, if there are two neighboring agents heading to the east and north, respectively, it is straightforward that the average direction is the northeast. But for the nonpolar or very weakly polar case, such as four neighboring agents heading to the east, north, west, and south, then what is the average direction? They are in a dilemma in deciding the average direction. Such situations happen when $\phi_{i}(k+1)=0$ or $\phi_{i}(k+1) \approx 0$, where the directions of individuals in agent $i$ 's neighborhood have no or an indistinct polarity. In such cases, the average directions are poorly defined. These cases are unavoidable in the DAR. Note that, in the case of $\phi_{i}(k+1)=0$ or $\phi_{i}(k+1) \approx 0$, it is not appropriate to stipulate the angle $\theta_{i}(k+1)$ of the complex zero-vector

$$
\frac{1}{n_{i}(k+1)} \sum_{j \in \Gamma_{i}(k+1)} e^{i \theta_{j}(k)}
$$

to be zero, which is equivalent to designating the east as the average direction. This stipulation favors a certain direction and thus breaks the rotationally symmetric property of the DAR in the swarm.

\section{ROLE OF SINGULARITIES IN THE EMERGENCE OF COLLECTIVE MOTION}

The singularity may be undesirable for theoretical analysis, but is tractable in numerical computation, for a computer has a certain numeric precision (floating-point representation) to express and compute numbers, and the average direction $\theta_{i}(k+1)$ can always be designated even when $\phi_{i}(k$ $+1)$ infinitely approaches zero. However, individuals do not know the average direction of motion when confronted with such singularity surroundings, but they are "forced in computer simulation to decide an average direction and move on accordingly. We will demonstrate that the singularity derived from this poorly defined case is favorable to the emergence of a swarm. This compelled mechanism tends to reduce the extreme local disorder of the system. The symmetry breaks at the singularity and a positive local polarity emerges from the isotropy.

In a weakly polar case [i.e., $\phi_{i}(k+1) \ll 1$ ], agents may still have some difficulty in determining the poorly defined average direction (see Fig. 3). Actually, a latent assumption embedded in the DAR is that every agent has an infinite ability to discriminate to find the right average direction $\theta_{i}(k+1)$ even under very small local polarity $\phi_{i}(k+1) \approx 0$. However, when the ability to discriminate is finite, the nearly singular case becomes a dilemma, namely, finding the average direction is a difficult task for the agents. It may be reasonable to suppose that for real agents, when $\phi_{i}(k+1)$ is larger than or equal to a certain threshold, say $\phi_{0}$, agent $i$ has the ability to determine the average direction, while when $\phi_{i}(k+1)<\phi_{0}$, agent $i$ is not able to detect the weak polarity direction. A smaller threshold $\phi_{0}$ corresponds to a higher demand upon the agents for the ability of polar discrimination. In a nearly locally isotropic case, an agent does not have enough justification to change its original direction. The sufficiency of the justification is parametrized as the threshold $\phi_{0}$. 


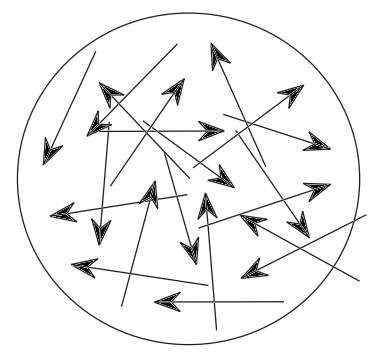

(a)

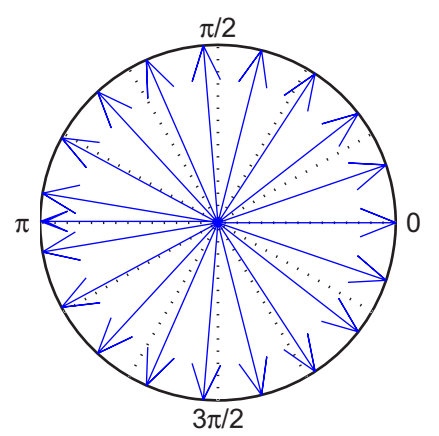

(c)

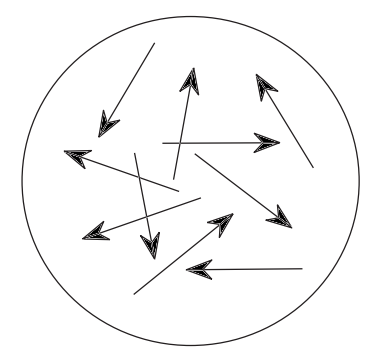

(b)

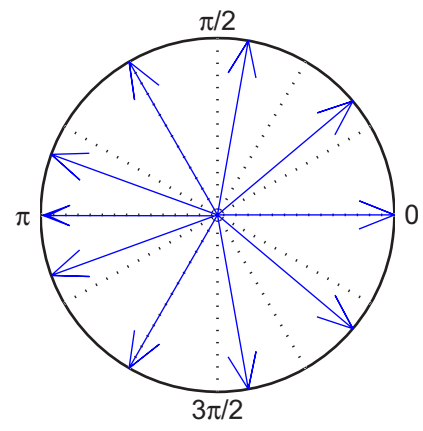

(d)
FIG. 3. (Color online) What the polarities $\phi=0.05$ and 0.1 look like. The arrows denote the unitary directional vectors. (a) $\phi$ $=0.05$. (b) $\phi=0.1$. (c) The directions of the 19 arrows that are uniformly distributed on the unit circle with one additional arrow pointing in the direction $\pi$, leading to $\phi=0.05$. (d) The directions of the nine arrows that are uniformly distributed with one additional arrow pointing in the direction $\pi$, leading to $\phi=0.1$. (a) and (b) were produced by translational movement of the arrows without changing the directions from the original figures (c) and (d), respectively.

To illustrate to what extent the symmetry-breaking effects at or near singularities contribute to the emergence of collective motion, we weaken the singularities in the AVM [26] and investigate the emergence of order again:

$$
\begin{gathered}
\vec{X}_{i}(k+1)=\vec{X}_{i}(k)+\phi_{i}^{\alpha}(k) e^{i \theta_{i}(k)} v_{0} \Delta t, \\
\phi_{i}(k+1)=\frac{1}{n_{i}(k+1)}\left|\sum_{j \in \Gamma_{i}(k+1)} e^{i \theta_{j}(k)}\right|, \\
\theta_{i}(k+1)= \begin{cases}\operatorname{angle}\left(\sum_{j \in \Gamma_{i}(k+1)} e^{i \theta_{j}(k)}\right), & \phi_{i}(k+1) \geq \phi_{0}, \\
\theta_{i}(k), & \phi_{i}(k+1)<\phi_{0},\end{cases} \\
i=1,2, \ldots, N ; \quad k=0,1,2, \ldots .
\end{gathered}
$$

Compared to Eq. (3), the only difference is that, for every agent $i$, when the local polarity $\phi_{i}(k+1) \geq \phi_{0}$, its next direction $\theta_{i}(k+1)$ is appointed as the average direction within its neighborhood; otherwise, it does not change its direction [33]. Note that, instead of adopting the previous value $\theta_{i}(k)$ here, $\theta_{i}(k+1)$ can also be assigned with a random direction

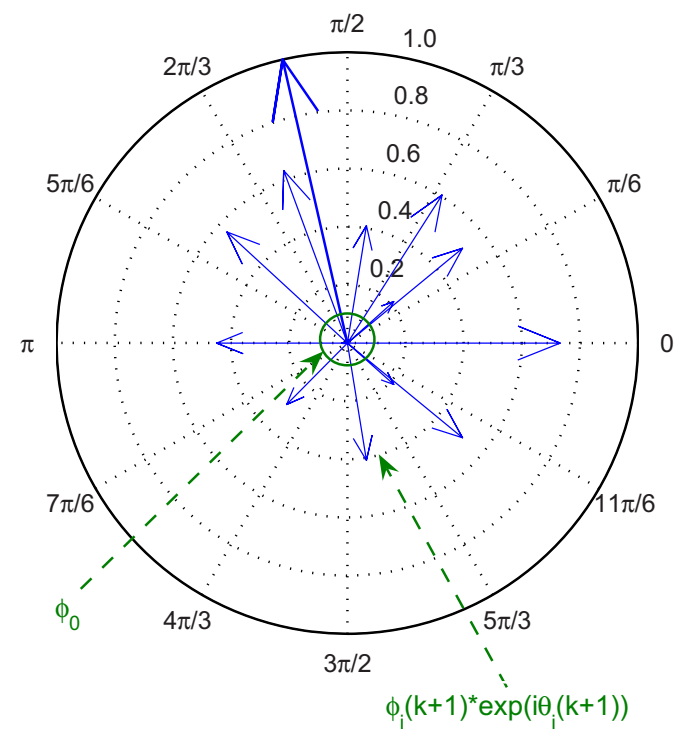

FIG. 4. (Color online) Illustration of the threshold $\phi_{0}$ for 2D swarm agents to update their directions of motion. The direction of agent $i$ is updated only when $\phi_{i}(k+1) \geq \phi_{0}$.

in the interval $[0,2 \pi)$, but should not be a specific direction such as the direction of the east or north, for this will introduce an additional symmetry-breaking factor to the swarm. Here the predefined constant $0 \leq \phi_{0} \leq 1$ serves as a threshold for agents to update their movement directions (see Fig. 4).

This adjustment in Eq. (10) weakens the singularities, but does not destroy the rotational-symmetry property of the model, that is, the model itself does not favor any predefined direction. When the threshold $\phi_{0}=0$, the singularityelimination model Eq. (10) degenerates to the standard AVM [26] as shown in Eq. (3). To measure the emergence of order within the swarm accurately, the standard deviation $S_{d}$ of the series of unitary directional vectors $e^{i \theta_{i}(k)}$ of all agents at time step $k$ is defined as follows:

$$
\psi(k)=\frac{1}{N} \sum_{i=1}^{N} e^{i \theta_{i}(k)} \in \mathrm{C},
$$

$$
S_{d}=\sqrt{\frac{1}{N} \sum_{i=1}^{N}\left[e^{i \theta_{i}(k)}-\psi(k)\right]\left[e^{i \theta_{i}(k)}-\psi(k)\right]^{*}} \in \mathbb{R} .
$$

The notation $*$ denotes the complex conjugate. $S_{d}$ reflects the degree of emergence in the swarm: when $\psi(k)=0$, that is, the swarm shows no polarity or emergence, we have

$$
S_{d}=\sqrt{\frac{1}{N} \sum_{i=1}^{N} e^{i \theta_{i}(k)}\left(e^{i \theta_{i}(k)}\right)^{*}}=1 .
$$

For the convergence case, i.e., direction consensus among all individuals, $|\psi(k)|=1$ and $S_{d}=0$. In numerical simulations, $S_{d} \ll 1$ reflects large-scale emergence, while $S_{d} \approx 1$ means isotropic dispersion.

The main concern is that the emergence of order displays a sharp transition. Figure 5(a) shows the unexpected behavior of $S_{d}$ as a function of $\phi_{0}$ in the steady state of the swarm: 

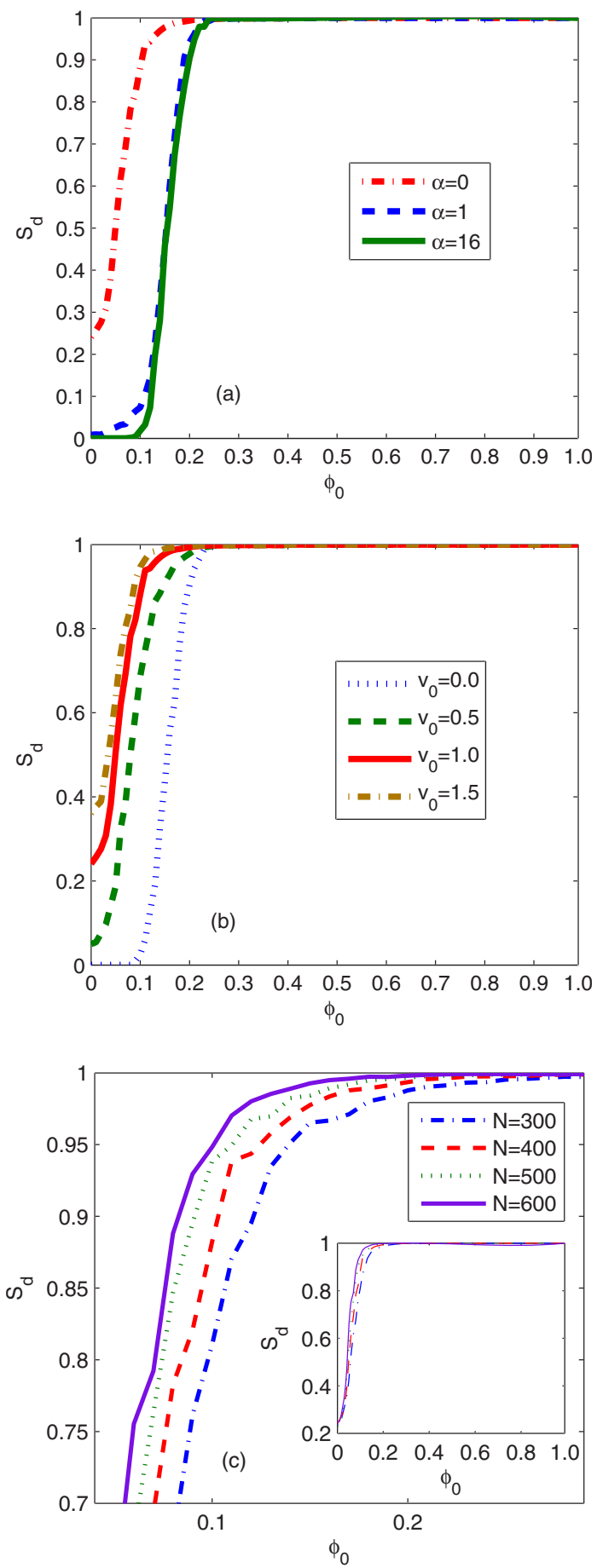

FIG. 5. (Color online) Oder emergence degree of 2D swarms. Consider $N$ agents moving in a 2D plane without boundary restrictions [26]. The positions of agents are initially randomly distributed on a disk of radius 2.5 with random initial directions. $R=2$. All estimates are the results of averaging over $M=400$ realizations of the swarm in steady state. The termination condition is that the standard deviation of $N$ vectors consisting of the coterminous directional differences of every agent is less than 0.0001. (a) Average standard deviation $S_{d}$ in steady state as a function of threshold $\phi_{0}$ $\in[0,1]$ for different $\alpha . N=400, v_{0}=1.0$. (b) $S_{d}$ in steady state as a function of threshold $\phi_{0}$ for different speeds $v_{0}$ with $\alpha=0$. $N=400$. (c) $S_{d}$ as a function of threshold $\phi_{0}$ for different densities with $v_{0}$ $=1.0$ and $\alpha=0$. for $\alpha=0$ and $\phi_{0}=0$, the swarm takes on large-scale order emergence; however, when the threshold $\phi_{0}$ increases a little, the degree of emergence of order reduces sharply. As the threshold $\phi_{0}$ continuously increases, an ordered state can never be achieved.

Of course, when the threshold $\phi_{0}$ is larger, the local polarity of every agent has less probability to exceed the threshold; therefore, the agents tend to move in their previous direction and the swarm will not gain order. ${ }^{1}$ The larger the threshold $\phi_{0}$, the fewer symmetry-breaking effects the swarm assumes, and thus the larger the degree of the symmetry property the swarm retains.

Figure 5(b) reports $S_{d}$ in steady state as a function of $\phi_{0}$ for different speeds $v_{0}$ with $\alpha=0$. The larger the speed parameter $v_{0}$, the larger the degree of symmetry the swarm retains. Figure 5(c) shows that, as the density of the swarm increases, the same degree of phase transition occurs for relatively smaller threshold values $\phi_{0}$.

The prolonged contacts among the agents can enhance the emergence of order, which is somewhat consistent with the results reported in Refs. [12,13,26,30]. This can also be seen from two aspects in Figs. 5(a) and 5(b). (i) From Fig. 5(a), for a certain threshold $\phi_{0}$, whether the threshold $\phi_{0}=0$ or not, the swarms with a larger $\alpha$ can generate more intensified emergence of order than when $\alpha=0$. For a larger $\alpha$, the speeds of agents are relatively slow in the transient process, especially at the onset of the evolution. Thus the transformations of the agents' positions are relatively less distinctive than in the case of $\alpha=0$, and the neighborhood relations tend to be retained in the next time step or even further. These prolonged contacts are beneficial to the directional consensus [26]. (ii) Figure 5(b) illustrates the difference in emergence for different $v_{0}$, and implies that a slower speed, which gives prolonged contacts among agents, enhances the emergence of order.

In Fig. 5(b), the speed $v_{0}=0$ represents a special case where all the agents in the swarm are still, with only their directions updating, and the neighborhood relations of every agent are invariant. Clearly, in this case, any value of $\alpha$ has no effect on the swarm. The curve of $\alpha=16\left(v_{0}=1.0\right)$ in Fig. 5(a) and the curve of $v_{0}=0.0$ in Fig. 5(b) are similar. Note that the speeds of all agents reach the maximum $v_{0} \neq 0$ after a short transient process [26]. We can conclude that the emergence of order is mainly determined by the early evolutional process.

For a smaller $\phi_{0}$, the demand of local polarity discrimination for swarm agents is higher. Also, for the same degree of emergence $S_{d}, \phi_{0}$ is larger in cases of $\alpha>0$ than when $\alpha=0$ [see Fig. 5(a)].

The singularity also has the property of instability and the swarm may sometimes be sensitive or even hypersensitive to

\footnotetext{
${ }^{1}$ When the threshold is larger, the swarm agents tend to show selectivity increasingly. They only interact with their neighbors in relatively strong polarity cases, and the influences of their neighbors in the less strong polarity cases are weakened. The agents tend to show more effect of "inertia" along their respective previous direction, and the symmetry property from the onset is thus more reserved.
} 
minor disturbance at or near singularities. For example, let $\varepsilon$ be infinitesimal. Then for the following two cases of the surroundings of agent $i$ at time step $k$ :

$$
\begin{gathered}
\sum_{j \in \Gamma_{i}(k+1)} \sin \left(\theta_{j}(k)\right)=0, \\
\sum_{j \in \Gamma_{i}(k+1)} \cos \left(\theta_{j}(k)\right) \rightarrow 0+\varepsilon \quad(\text { case } 1), \\
\sum_{j \in \Gamma_{i}(k+1)} \sin \left(\theta_{j}(k)\right)=0, \\
\sum_{j \in \Gamma_{i}(k+1)} \cos \left(\theta_{j}(k)\right) \rightarrow 0-\varepsilon \quad(\text { case } 1),
\end{gathered}
$$

the corresponding results are

$$
\theta_{i}(k+1)=\arctan \left(\frac{0}{0+\varepsilon}\right)
$$

and

$$
\theta_{i}(k+1)=\arctan \left(\frac{0}{0-\varepsilon}\right),
$$

respectively, which differ by $\pi$; this difference is sharp and unexpected. Figure 6(a) gives an illustration of three neighboring agents near a singular case. Agents 1 and 3 move along the directions of 0 and $2 \pi / 3$, respectively. Suppose that the direction of agent 2 is $\left(4 \pi / 3-\xi_{1}\right)$ before the disturbance, where $\xi_{1}$ is infinitesimal; then the coherent movement direction is in the second quadrant of the 2D plane, i.e.,

$$
\theta_{1}(k+1)=\theta_{2}(k+1)=\theta_{3}(k+1) \in(\pi / 2, \pi) .
$$

When agent 2 is subjected to a minor disturbance and changes its direction to $\left(4 \pi / 3+\xi_{2}\right)$ [denoted as agent $2^{\prime}$ in Fig. 6(a)], where $\xi_{2}$ is also infinitesimal, then

$$
\theta_{1}(k+1)=\theta_{2}(k+1)=\theta_{3}(k+1) \in(3 \pi / 2,2 \pi),
$$

that is, the movement direction is in the fourth quadrant. The results in these two cases differ by at least $\pi / 2$. Similar phenomena of disturbance sensitivity exist even for large swarms from a macroscopic perspective [Fig. 6(b)]. The final emergent polarity of the whole system is robust to small disturbances in some cases, but it can also be extremely fragile under some circumstances. Even for the same swarm with the same initial conditions, different levels of precision in numerical simulations will result in different emergent directions of the swarm. Anticipation of the result may not be possible, unlike with the linearity or quasilinearity model of the swarm (see Appendixes A and B). Such a robust, yet fragile (or vulnerable) property is also found in many networks [32], and this may be one of the universal properties of complex systems. Modern analysis and technologies typically think much of robustness instead of fragility. However, in our opinion, the latter deserves more attention in the study of multiagent systems. It is this singularity that partially endows the system with the significant nonlinear characteristics.
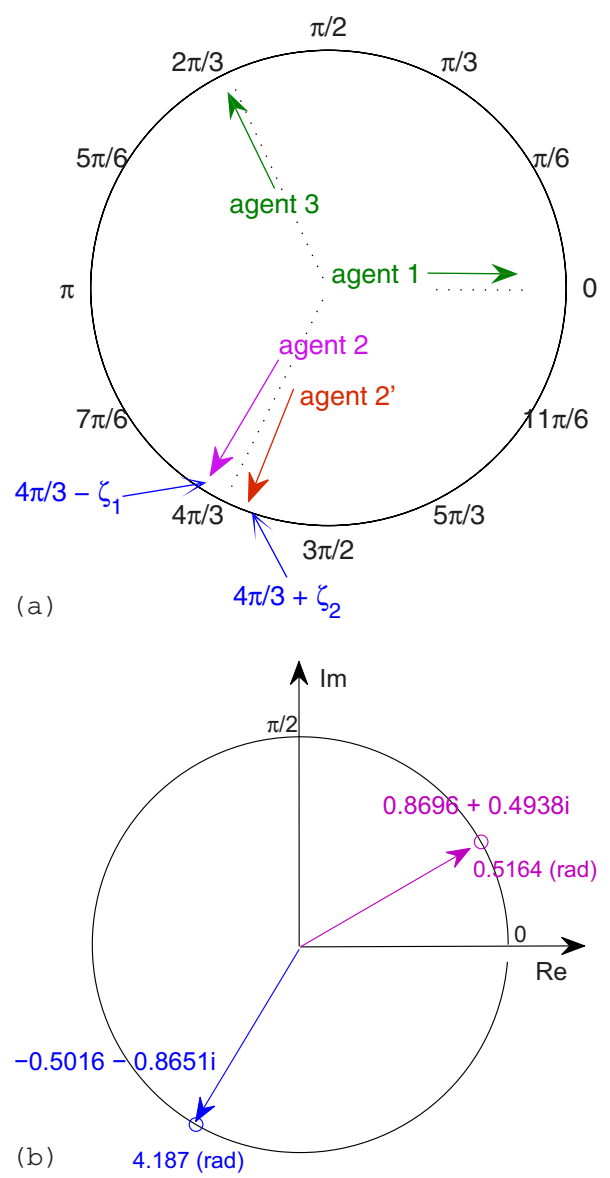

FIG. 6. (Color online) (a) Illustration of the singularity of three neighboring agents in the 2D plane. (b) Abrupt change induced by minor directional disturbance of only one agent in an approximately isotropic swarm in 2D space. In this instance, all agents are randomly distributed on a disk with random radii distributed in the interval $[0,2.5)$ and random direction angles distributed in the interval $[0,2 \pi) . N=500, R=3.0, v_{0}=1.0$, and $\alpha=0$. The final coherent movement direction is $4.187 \mathrm{rad}$, as illustrated in the third quadrant on the unit circle in the 2D complex plane, but when an agent (numbered 474 of this example) in the initial condition changes its direction by only 0.0001 rad counterclockwise, the coherent movement direction of the swarm changes to $0.5164 \mathrm{rad}$.

\section{CONCLUSIONS}

For swarms of homogeneous agents, emergence of order can be generated by a simplistic mechanism for the agentsdoing what their near neighbors do. This mechanism seems to be symmetric without any preferred direction(s). But when referenced coordinates are introduced into such swarm systems to denote and compute the directions, the rotationally symmetric DAR actually has symmetry-breaking effects (in contrast, the linear or quasilinear form of the DAR is itself a symmetry-breaking rule with a certain preferred direction). It can designate the average direction of agents in the surroundings of a polarity that is not apparent, or even a singularity. This plays an important role in promoting large-scale emergence of order, especially from the isotropic state.

The DAR may be suitable for self-propelled physical particles such as the atoms in a ferromagnet, whose mechanism 
of directional determination may be precise. But is the DAR [with or without other additional rule(s)] suitable for biological bacterial, fish, or bird swarms? The swarm agents are supposed to be able to detect the average directions even at nonapparent local polarities for large-scale emergence of order, but how do they discriminate those local polarities around themselves that are even smaller than a very small threshold $\phi_{0}$ (see Fig. 3)? In addition, do the swarm agents have their private referenced coordinate(s) as we humans assign to them [such as the case of Fig. 6(a)]? If each of them does have its private coordinates, how do the agents calibrate their private reference coordinates to the common "standard" one? Of course, it is not necessarily the case, as some species do have references (such as the magnetic field of earth, the air or ocean current), but others do not.

Therefore, thus far, we may not have completely satisfactory answers to the questions (i) why the large-scale emergence of order is generated in natural swarms without any preferred direction; and (ii) what symmetry-breaking rule(s) the natural swarms actually adopt. There are some deep secrets in natural swarm intelligence that we humans have not discovered yet. The exploration of nature's secrets is an interesting yet challenging journey ahead.

\section{ACKNOWLEDGMENTS}

We wish to acknowledge Dr. Iain D. Couzin, who offered many valuable suggestions and comments for the early version of this paper. H.T.Z. acknowledges NNSFC Grant No. 60704041 and NSF-HUST Grant No. 2006Q041B; T.Z. acknowledges NNSFC Grant No. 10635040 and 973 Project No. 2006CB705500.

\section{APPENDIX A: LINEAR SCALAR EXPRESSION OF THE DAR}

Let $\theta_{i}(0)$ be the scalar movement direction of agent $i$ in polar coordinates at initial time step $k=0, i=1,2, \ldots, N$; these initial directions $\theta_{i}(0)$ are evenly distributed in the interval $[0,2 \pi)$ or $(0,2 \pi]$. The DAR that every agent moves toward the average movement direction of its near neighbors is expressed in the linear scalar form as follows $[12,30]$ :

$$
\theta_{i}(k+1)=\frac{1}{n_{i}(k+1)}\left(\sum_{j \in \Gamma_{i}(k+1)} \theta_{j}(k)\right),
$$

where $\theta_{i}(k+1)$ is the average direction of agent $i$ 's near neighbors (including itself) at the next time step $k+1$.

Here we show that Eq. (A1) is a symmetry-breaking rule in itself, which has one certain preferred direction for the swarms.

Note that the convex combination is defined as follows: for the non-negative parameters $\gamma_{i}, i=1,2, \ldots, N$, with constraint $\sum_{i=1}^{N} \gamma_{i}=1$, the sum

$$
x=\sum_{i=1}^{N} \gamma_{i} x_{i}
$$

is a convex combination of the $N$ variables $x_{i}, i$ $=1,2, \ldots, N$. The convex combination has the property

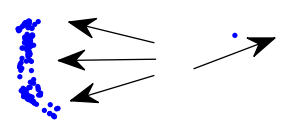

(a)

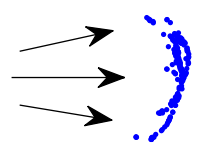

(c)

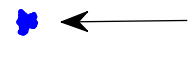

(b)

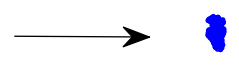

(d)
FIG. 7. (Color online) Illustration of the existence of the preferred directions of the linear scalar rule Eq. (A1). The common simulation parameters of the swarm are $N=200, L=5$. (a) Nonconvergence case, $\theta \in[0,2 \pi), R=0.5$, and $v_{0}=0.5$. (b) Convergence case, $\theta \in[0,2 \pi), R=1.0$, and $v_{0}=0.5$. (c) Nonconvergence case, $\theta \in[-\pi, \pi), R=0.5$, and $v_{0}=0.5$. (d) Convergence case, $\theta$ $\in[-\pi, \pi), R=1.0$, and $v_{0}=0.5$. In the cases (a),(b), the preferred directions are both $\pi$; in (c),(d), the preferred directions are 0 in polar coordinates.

$\min \left\{x_{i}\right\} \leq x_{i} \leq \max \left\{x_{i}\right\}$ for all $i=1,2, \ldots, N$. When all $\gamma_{i}$ $>0, i=1,2, \ldots, N, x$ is a strictly convex combination of $x_{i}, \quad i=1,2, \ldots, N$ and $\min \left\{x_{i}\right\}<x_{i}<\max \left\{x_{i}\right\}$.

We show that the scalar rule Eq. (A1) with the initial condition $\theta_{i}(0) \in[0,2 \pi), i=1,2, \ldots, N$ favors the movement direction $\pi$. In Eq. (A1), the average direction $\theta_{i}(k+1)$ is a convex combination of the $n_{i}(k+1)$ elements $\theta_{j}(k)$ with equal coefficients

$$
\gamma_{1}(k+1)=\gamma_{2}(k+1)=\cdots=\gamma_{j}(k+1)=\cdots=1 / n_{i}(k+1)
$$

for all $j \in \Gamma_{i}(k+1)$. Therefore, in Eq. (A1), the initial conditions $\theta_{i}(0) \in[0,2 \pi)$ imply that $\theta_{i}(k) \in[0,2 \pi)$ for all $k>0$. Denoting the set of the directions of all agents as $\{\theta(k)\}$ at time step $k$, the convex hull $\operatorname{conv}\{\theta(k)\}$ satisfies

$$
\operatorname{conv}\{\theta(k+1)\} \subseteq \operatorname{conv}\{\theta(k)\}
$$

for all $k$, i.e., the convex hull is nonincreasing as the system evolves. Since the initially distributed directions of agents are random and statistically symmetric, the convex hull does not favor any interval $[0, \pi)$ or $[\pi, 2 \pi)$ statistically. Generally, the convex hull $\operatorname{conv}\{\theta(k)\}$ decreases as the system evolves. When it reduces to a singleton $\left\{\theta_{0}\right\}$ (i.e., a point set of one element $\theta_{0}$ ), we say that the system converges to the direction $\theta_{0}$. For the nonconvergence case, the snapshot of motions of the system always looks like Fig. 7(a). For the convergence case, the convex hull converges to a singleton $\{\pi\}$ with little error [see Fig. 7(b)].

Further, suppose $\theta_{i}(0) \in[-\pi, \pi) \quad$ [instead of $\theta_{i}(0)$ $\in[0,2 \pi)$ as above $]$ for all agents $i$ in the swarm Eq. (A1); then generally the motion of the system looks like Figs. 7(c) and $7(\mathrm{~d})$. The agents in this case favor the movement direction 0. Actually, for the linear scalar Eq. (A1) with $\theta_{i}(0) \in[-\pi+\alpha, \pi+\alpha)$, where $\alpha \in[0,2 \pi)$, the final polarity 


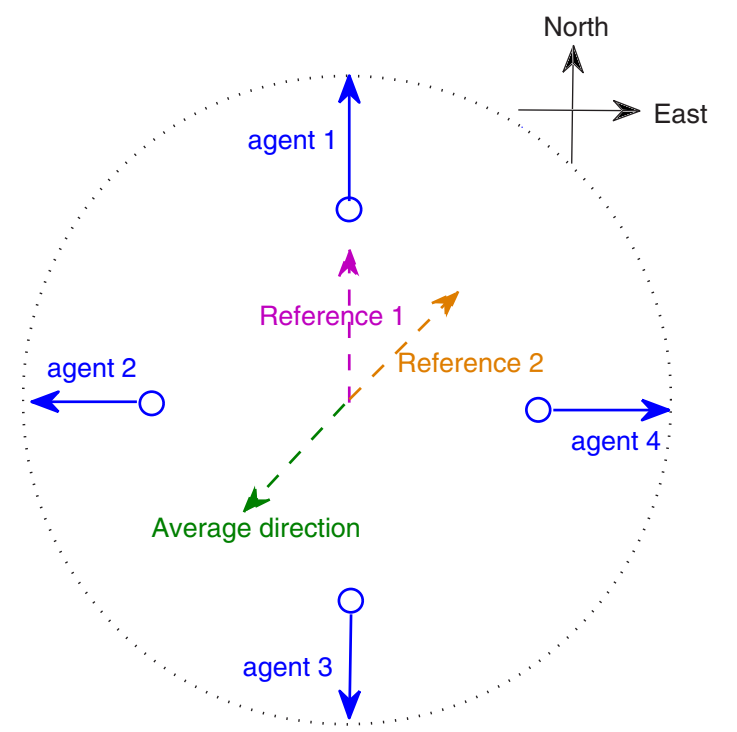

FIG. 8. (Color online) Let $\theta_{i}(0) \in[0,2 \pi), i=1,2, \ldots, N$, under all possible scalar reference coordinates. The directions of agents under any reference coordinate are counted counterclockwise. The average direction of the four neighboring agents is shown in this figure under reference coordinate 1 or 2 . For the cases under all possible reference coordinates, see Table I. Note that, for these four agents, the directions can be viewed as evenly distributed in the interval $[0,2 \pi)$, with average direction $\pi$, only when the direction of the reference coordinate points west (east) by south (north), $\pi / 4$.

direction and convergence direction of the swarm can both be predicted as the direction $\alpha$. This is equivalent to the following: the property of the preferred direction of the system Eq. (A1) is reference-coordinate dependent. The reference-coordinate dependence is also illustrated in Fig. 8.

The linear scalar rule Eq. (A1) also has the property that, under this rule, the directions in the interval $[0,2 \pi$ ) (or any other interval of the initial directional distribution) do nothave equal (or rotationally symmetric) "status." Here we illustrate that this nonsymmetric linear scalar rule serves as the very interpretation of the undesired phenomenon mentioned at the end of $[12,30]$. As stated in Refs. $[12,30]$, it is straightforward that the two directions 0.1 and $(2 \pi-0.1)$ are obviously very close in polar coordinates on the plane [see Fig. 9(a)], the average direction is the direction of the east. But the average direction is actually $\pi$, according to Eq. (A1),

TABLE I. The average direction of the four agents in Fig. 8 under all possible scalar reference coordinates. (East, north] means that the direction of the reference coordinate is located in the range from east counterclockwise to north but not including east. The average direction shifts discretely.

\begin{tabular}{ll}
\hline \hline Reference direction & Average direction \\
\hline (East, north] & West by south $\pi / 4$ \\
(North, west] & East by south $\pi / 4$ \\
(West, south] & East by north $\pi / 4$ \\
(South, east] & West by north $\pi / 4$ \\
\hline \hline
\end{tabular}

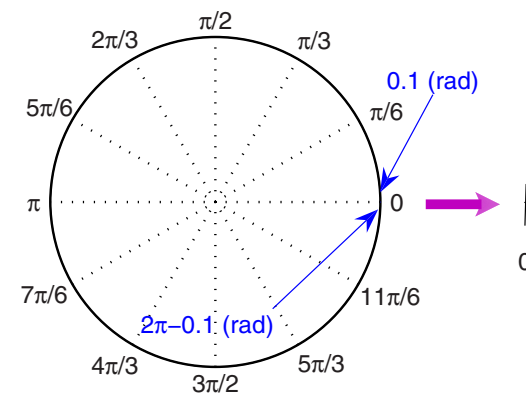

(a)

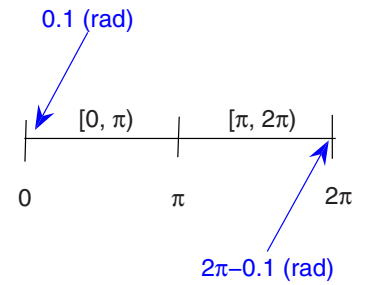

(b)

FIG. 9. (Color online) Illustration of the non-rotationallysymmetric relationship of the directions' adjacency property. The directions that lie at both ends of the interval $[0,2 \pi)$, such as 0.1 and $(2 \pi-0.1)$, should be very close directions for swarm agents in the $2 \mathrm{D}$ plane by intuition. However, they are actually not considered as close under the linear scalar rule Eq. (A1); the average direction is $\pi$ instead of 0 (the direction of the east). This is the very reason for the counterintuitive phenomenon mentioned in the literature $[12,30]$, which cannot be overcome in linear models.

so both of the two agents will almost reverse compared to their former directions 0.1 and $(2 \pi-0.1)$, respectively. Actually, the two directions are not considered as adjacent in the scalar computation rule Eq. (A1). This can be clearly seen in Fig. 9(b); the adjacency relationship of the two directions 0.1 and 0.3 is not the same as that of $(2 \pi-0.1)$ and 0.1 . This symmetry-breaking status is the very reason why some undesired problems arise, as mentioned in the literature $[12,30]$.

The predictable polarity together with the counterintuitive and undesired problems reflect the loss of the rotationally symmetric property of the DAR after linearity as in Eq. (A1).

\section{APPENDIX B: QUASILINEAR EXPRESSION OF THE DAR}

The restriction of the principal value interval $(-\pi / 2, \pi / 2)$ of the $\operatorname{arctangent}$ function $\arctan (\cdot)$ in Ref. [13] reduces the essentially nonlinear directional alignment model [Eqs. (3) and (5)] to a quasilinear one. Note that, for the principal value interval $(-\pi / 2, \pi / 2)$, the tangent and arctan-

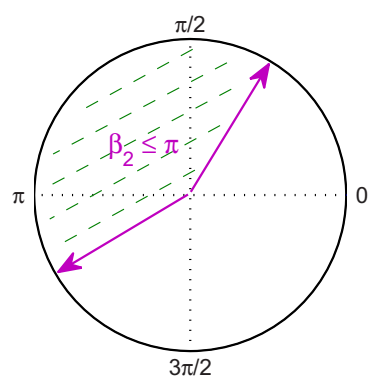

(a)

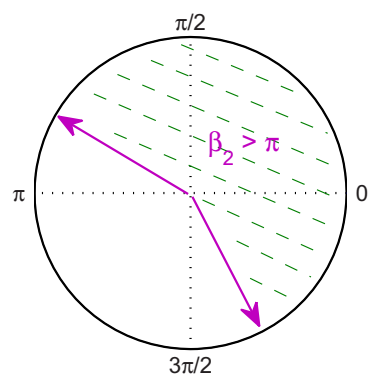

(b)
FIG. 10. (Color online) Illustration of motion direction range of agents. (a) Less and (b) more than the half plane. 
gent functions are both monotropic. Therefore, from Eq. (5) in Sec. III, we have

$$
\tan \left(\theta_{i}(k+1)\right)=\frac{\sum_{j \in \Gamma_{i}(k+1)}\left[\cos \left(\theta_{j}(k)\right) \tan \left(\theta_{j}(k)\right)\right]}{\sum_{j \in \Gamma_{i}(k+1)} \cos \left(\theta_{j}(k)\right)} .
$$

In the quasilinear expression Eq. (B1) of the DAR, the function $\tan \left(\theta_{i}(k+1)\right)$ can be viewed as a strict and linear convex combination of the $n_{i}(k+1)$ series of functions $\tan \left(\theta_{j}(k)\right)$ with the corresponding positive coefficients

$$
\frac{\cos \left(\theta_{j}(k)\right)}{\sum_{j \in \Gamma_{i}(k+1)} \cos \left(\theta_{j}(k)\right)}>0
$$

for all $j \in \Gamma_{i}(k+1)$.

As a consequence, the angle $\theta_{i}(k+1)=\arctan \left(\tan \theta_{i}(k\right.$ $+1)$ ) with its range $\theta_{i}(k+1) \in(-\pi / 2, \pi / 2)$. That is, for the initial directional distribution $\theta_{i}(0) \in(-\pi / 2, \pi / 2)$, $i=1,2, \ldots, N$, all agents under this quasilinearity rule only move toward directions in the right half plane at any iteration. In this case, $\phi_{i}(k+1)>0$ always strictly holds for any agent $i$ and at any time step $k$; thus no singularity exists in this quasilinear swarm. As in the linear form, the swarm in this case prefers the motion direction 0 statistically [for illustrations, see Figs. $7(\mathrm{c})$ and $7(\mathrm{~d})$.

Actually, for any interval $\left(\beta_{1}, \beta_{1}+\beta_{2}\right)$ defined for the initial movement direction $\theta_{i}(0)$ of agents $i, i=1,2, \ldots, N, \beta_{1}$ $\in \mathrm{R}$, and with the range of the arctangent function $\arctan (\cdot)$ also defined in the same interval $\left(\beta_{1}, \beta_{1}+\beta_{2}\right)$, we have the following. (1) When the parameter $\beta_{2}$ satisfies $0<\beta_{2} \leq \pi$, that is, in less than the half plane [see Fig. 10(a)], the system Eq. (3) or Eq. (5) has a quasilinear property without any possibility of singularities, and $\phi_{i}(k+1)>0$ always holds for all $i$ and $k$. (2) When $\beta_{2}>\pi$, that is, in more than the half plane [see Fig. 10(b)], there exist the possibilities that $\phi_{i}(k$ $+1)=0$ [see also Eq. (9) in Sec. III], and the system is strongly nonlinear with singularities, but it still cannot be viewed as a rotationally symmetric rule when $\beta_{2}<2 \pi$.
[1] J. K. Parrish, Science 284, 99 (1999).

[2] P. K. Visscher, Nature (London) 421, 799 (2003).

[3] H. Levine, W.-J. Rappel, and I. Cohen, Phys. Rev. E 63, 017101 (2000).

[4] I. D. Couzin, J. Krause, R. James, G. D. Ruxton, and N. R. Franks, J. Theor. Biol. 218, 1 (2002).

[5] I. D. Couzin, J. Krause, N. R. Franks, and S. A. Levin, Nature (London) 433, 513 (2005).

[6] J. K. Parrish, S. V. Viscido, and D. Grunbaum, Biol. Bull. 202, 296 (2002).

[7] J. Buhl, D. J. T. Sumpter, I. D. Couzin, J. J. Hale, E. Despland, E. R. Miller, and S. J. Simpson, Science 312, 1402 (2006).

[8] M. R. D’Orsogna, Y. L. Chuang, A. L. Bertozzi, and L. S. Chayes, Phys. Rev. Lett. 96, 104302 (2006).

[9] D. Grunbaum, Science 312, 1320 (2006).

[10] C. M. Topaz, A. L. Bertozzi, and M. A. Lewis, Bull. Math. Biol. 68, 1601 (2006).

[11] A. Kolpas, J. Moehlis, and I. G. Kevrekidis, Proc. Natl. Acad. Sci. U.S.A. 104, 5931 (2007).

[12] A. Jadbabaie, J. Lin, and A. S. Morse, IEEE Trans. Autom. Control 48, 988 (2003).

[13] L. Moreau, IEEE Trans. Autom. Control 50, 169 (2005).

[14] D. J. Low, Nature (London) 407, 465 (2000).

[15] D. Helbing, I. Farkas, and T. Vicsek, Nature (London) 407, 487 (2000).

[16] Z. Neda, E. Ravasz, Y. Brechet, T. Vicsek, and A. L. Barabasi, Nature (London) 403, 849 (2000).

[17] V. Narayan, S. Ramaswamy, and N. Menon, Science 317, 105 (2007).
[18] M. van Hecke, Science 317, 49 (2007).

[19] K. E. Parsopoulos and M. N. Vrahatis, Nat. Comput. 1, 235 (2002).

[20] J. H. Holland, Adaptation in Nature and Artificial Systems (MIT Press, Cambridge, MA, 1992).

[21] T. Vicsek, A. Czirók, E. Ben-Jacob, I. Cohen, and O. Shochet, Phys. Rev. Lett. 75, 1226 (1995).

[22] G. Gregoire and H. Chate, Phys. Rev. Lett. 92, 025702 (2004).

[23] C. Huepe and M. Aldana, Phys. Rev. Lett. 92, 168701 (2004).

[24] M. Aldana, V. Dossetti, C. Huepe, V. M. Kenkre, and H. Larralde, Phys. Rev. Lett. 98, 095702 (2007).

[25] M. Nagy, I. Daruka, and T. Vicsek, Physica A 373, 445 (2007).

[26] W. Li and X. Wang, Phys. Rev. E 75, 021917 (2007).

[27] J. Toner and Y. Tu, Phys. Rev. Lett. 75, 4326 (1995).

[28] J. Toner and Y. Tu, Phys. Rev. E 58, 4828 (1998).

[29] T. Vicsek, Nature (London) 411, 421 (2001).

[30] A. V. Savkin, IEEE Trans. Autom. Control 49, 981 (2004).

[31] It is easy to illustrate the undesired phenomenon of the linearity rule. Suppose that there are two neighboring agents heading east and west, respectively, for the direction domain $[0,2 \pi)$. The average direction of these agents is $\pi / 2$; however, for the direction domain $(0,2 \pi]$, the average direction is $3 \pi / 2$. See also Appendix A.

[32] R. Albert, H. Jeong, and A. L. Barabási, Nature (London) 406, 378 (2000).

[33] Such a direction assignment can be found implicitly in some prior swarm models $[4,5]$. 